September 6, 2018

MPI-Ph/93-63

LMU-TPW 1993-23

\title{
Unitary Continuous Representations of Compact Quantum Groups
}

\author{
Bernhard Drabant and Wolfgang Weich \\ Max-Planck-Institut für Physik, Föhringer Ring 6, D-80805 München \\ Sektion Physik der Universität München, Theresienstraße 37, D-80333 München
}

\begin{abstract}
Generalizing the notion of continuous Hilbert space representations of compact topological groups we define unitary continuous correpresentations of $C^{*}$-completions of compact quantum group Hopf algebras on arbitrary Hilbert spaces. It is proved that the unitary continuous correpresentations decompose in finite dimensional irreducible correpresentations.
\end{abstract}

\section{Introduction}

Continuous Symmetries play a fundamental rôle in physics. In quantum mechanics the symmetries are represented by unitary operators on the Hilbert space of states which is in general infinite dimensional. This is the physical motivation to study the unitary continuous representations of topological groups. With the quantum groups a more general concept of symmetry has been introduced into physics [1, 2, 6]. They are not any more sets of transformations but are described by non-commutative non-cocommutative $*$-Hopf algebras which should be understood in some sense as the polynomials over the fictive quantum group. Unitary finite dimensional correpresentations

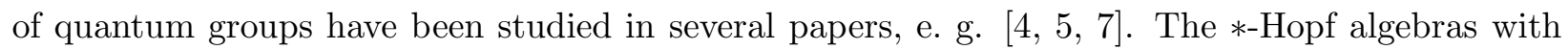
$C^{*}$-norms can be completed to $C^{*}$-algebras thus describing non-commutative topological spaces [6]

- in the case of commutative $*$-Hopf algebras the $C^{*}$-completions are the algebras of continuous functions over compact topological groups. For an application in quantum mechanics one is now interested in the unitary continuous correpresentations on arbitrary Hilbert spaces of those $C^{*}$ algebras arising from non-commutative non-cocommutative $*$-Hopf algebras.

In section 2 correpresentations of $*$-Hopf algebras and the theory of $*$-Hopf algebras of compact quantum groups are reviewed [4, 6]. With the help of the Haar functional on a $*$-Hopf algebra of a compact quantum group the Hilbert space completion is introduced in section 3 . The left action by multiplication yields a $C^{*}$-norm. These concepts are used in section 4 to define the continuous correpresentations of the $C^{*}$-completion. In the concluding theorem it is stated that 
the unitary continuous correpresentations on any Hilbert space decomposes into finite dimensional correpresentations of the underlying $*$-Hopf algebra. This is a generalization of the classical results on unitary representations of compact topological groups [3].

\section{The compact quantum group algebra $\mathcal{A}$}

In this section definitions and results of [4, 6] are quoted. Henceforth $\mathcal{A}$ is a $*$-Hopf algebra with comultiplication $\Delta: \mathcal{A} \rightarrow \mathcal{A} \otimes \mathcal{A}$, counit $\epsilon: \mathcal{A} \rightarrow \mathbb{C}$ and antipode $S: \mathcal{A} \rightarrow \mathcal{A}$.

For some vector space $V$ let $\operatorname{End}(V)$ denote its endomorphisms. The transposition operator $\sigma_{V}$ acts as endomorphism on $V \otimes V$ by $a \otimes b \mapsto b \otimes a$.

2.1 Definition. A correpresentation $(T, V)$ of $\mathcal{A}$ on the vector space $V$ is given by

(i) $T \in \mathcal{A} \otimes \operatorname{End}(V)$, such that

(ii) $\quad T_{13} T_{23}=(\Delta \otimes i d)(T), T_{23}:=1_{\mathcal{A}} \otimes T, T_{13}:=\left(\sigma_{\mathcal{A}} \otimes i d\right)\left(T_{23}\right)$,

(iii) $\quad(\epsilon \otimes i d)(T)=1_{\operatorname{End}(V)}$.

If $V$ is a Hilbert space the correpresentation $(T, V)$ of $\mathcal{A}$ is called unitary if $T^{*} T=1_{\mathcal{A}} \otimes 1_{\operatorname{End}(V)}=$ $T T^{*}$, where $T^{*}:=\left({ }^{*} \otimes^{*}\right)(T)$.

If $\operatorname{dim}(V)=n<\infty$ one can choose a basis $\left\{e_{i}\right\}_{i=1, . ., n}$ and a dual basis $\left\{e_{i}^{*}\right\}_{i=1, . ., n}$ with $e_{i}^{*}\left(e_{j}\right)=\delta_{i j}$. One can expand $T=\sum_{i, j=1}^{n} t_{i j} \otimes\left(e_{i} \otimes e_{j}^{*}\right)$ and obtains in particular $\Delta\left(t_{i j}\right)=\sum_{k} t_{i k} \otimes t_{k j}$ and $\epsilon\left(t_{i j}\right)=\delta_{i j}$. If $V$ is a Hilbert space, $(T, V)$ a unitary correpresentation, then $e_{i}^{*}=\left(e_{i} \mid\right.$.) and $S\left(t_{i j}\right)=t_{j i}^{*}$.

2.2 Definition. Let $(T, V)$ be a correpresentation of $\mathcal{A}$ on the vector space $V$. A linear subspace $W$ of $V$ is called invariant if $T\left(1_{\mathcal{A}} \otimes W\right) \subset \mathcal{A} \otimes W$.

$(T, V)$ is called irreducible if $V$ and $\{0\}$ are the only invariant subspaces of $V$.

Let $(S, W)$ be another correpresentation of $\mathcal{A}$. A linear operator $L: V \rightarrow W$ is called an intertwiner if $S\left(1_{\mathcal{A}} \otimes L\right)=\left(1_{\mathcal{A}} \otimes L\right) T$.

$(T, V)$ and $(S, W)$ are called equivalent if there exists a bijective intertwiner.

2.3 Schur's Lemma. Let $L: V \rightarrow W$ be an intertwiner for the correpresentations $(T, V)$ and $(S, W)$ of $\mathcal{A}$. If both are irreducible then either $L=0$ or $L$ is bijective.

2.4 Definition. The collection of all equivalence classes of irreducible unitary finite dimensional correpresentations of $\mathcal{A}$ is called $\hat{\mathcal{A}}$.

Any $*$-Hopf algebra has in particular the scalar unitary correpresentation $\left(1_{\mathcal{A}}, \mathbb{C}\right)$. We denote its equivalence class by $\hat{0} \in \hat{\mathcal{A}}$. For each $\alpha \in \hat{\mathcal{A}}$ we choose a representative $\left(T^{\alpha}, V^{\alpha}\right)$ which is unitary. 
2.5 Proposition. For each $\alpha \in \hat{\mathcal{A}}$ let $\left\{t_{i j}^{\alpha}\right\}_{i, j}$ be the set of matrix elements of $\left(T^{\alpha}, V^{\alpha}\right)$. Then $\left\{t_{i j}^{\alpha}\right\}_{\alpha \in \hat{\mathcal{A}}, i, j}$ is a linear independent set.

Setting $\mathcal{A}^{\alpha}:=\operatorname{span}\left\{t_{i j}^{\alpha}\right\}_{i j} \subset \mathcal{A}$ for $\alpha \in \hat{\mathcal{A}}$ the following definition is motivated by the theory of compact topological groups.

2.6 Definition. $\quad A *$-Hopf algebra $\mathcal{A}$ is a compact quantum group algebra if it is the linear span of the matrix elements of its unitary irreducible finite-dimensional correpresentations: $\mathcal{A}=\bigoplus_{\alpha \in \hat{\mathcal{A}}} \mathcal{A}^{\alpha}$.

The algebra of characteristic functions on a compact topological group is such a compact quantum group algebra. The Hopf algebras of the quantum groups $S U_{q}(N)$ and $S O_{q}(N)(q>0)$ of [2] are non-commutative examples.

Having a decomposition of $\mathcal{A}$ the Haar functional can be defined.

2.7 Definition. The linear functional $h: \mathcal{A} \rightarrow \mathbb{C}$ with $h\left(1_{\mathcal{A}}\right)=1$ and $h_{\left.\right|_{\mathcal{A}} ^{\alpha}}=0$ for $\alpha \neq \hat{0}$ is called the Haar functional.

2.8 Theorem. The Haar functional $h$ on the compact quantum group algebra $\mathcal{A}$ is uniquely determined by its properties

$$
(h \otimes i d)(\Delta(a))=h(a) 1_{\mathcal{A}}=(i d \otimes h)(\Delta(a)) \quad \text { and } \quad h\left(1_{\mathcal{A}}\right)=1 .
$$

The Haar functional is positive: $h\left(a^{*} a\right)>0$ if $a \neq 0$.

\section{The completions of $\mathcal{A}$}

In sections 3. and 4. $\mathcal{A}$ is always understood to be a compact quantum group algebra with Haar functional $h$. This Haar functional defines an inner product $\mathcal{A} \times \mathcal{A} \ni(a, b) \mapsto(a \mid b):=h\left(a^{*} b\right)$ which makes $\mathcal{A}$ a pre-Hilbert space. Thus also $\mathcal{A} \otimes \ldots \otimes \mathcal{A}$ becomes naturally a pre-Hilbert space. The Hilbert space completion of the pre-Hilbert spaces $X$ and $Y$ is denoted by $X \hat{\otimes} Y$, the Hilbert space completion of the direct sum of the pre-Hilbert spaces $X_{i}, i \in I$ is denoted by $\hat{\oplus}_{i \in I} X_{i}$. For a normed vector space $V$ we denote its continuous endomorphisms with $\mathrm{B}(V)$.

3.1 Definition. For each $n \in \mathbb{I}$ let $\ell^{\hat{\otimes} n}$ be the Hilbert space completion of the $n$-fold tensor product of $\mathcal{A}$, let $\ell^{\hat{\otimes} 0}:=\mathbb{C}$.

$$
\mathbf{L}:=\bigoplus_{n \in N_{0}}^{\wedge} \ell^{\hat{\otimes} n} .
$$

$\ell:=\ell^{\otimes} 1$ corresponds to the square integrable functions over the quantum group.

$\mathcal{A}$ acts on itself by left multiplication. This yields a $*$-representation $\mathcal{A} \ni a \mapsto \lambda(a) \in \mathrm{B}(\mathcal{A})$, $\lambda(a): b \mapsto a b \in \mathcal{A}$. Because of the unitarity condition $\sum_{i} t_{i j}^{\alpha *} t_{i k}^{\alpha}=\delta_{j k}$ in $\mathcal{A}^{\alpha}$ one gets that 
$\lambda(a)$ is a bounded operator on the pre-Hilbert space $\mathcal{A}$ and can therefore be extended to $\ell$, i. e. $\mathcal{A} \ni a \mapsto \lambda(a) \in \mathrm{B}(\ell)$ is a faithful Hilbert space representation of $\mathcal{A}[$ 国. Counit and comultiplication are *-algebra homomorphisms and serve to define Hilbert space representations of $\mathcal{A}$ on $\ell^{\hat{\otimes} n}$ and L.

3.2 Definition. $\Delta^{(0)}:=\epsilon$. For $n>0, \Delta^{(n)}:=\left(\Delta^{(n-1)} \otimes i d\right) \circ \Delta . \Lambda^{(n)}:=\lambda^{\otimes n} \circ \Delta^{(n)}: \mathcal{A} \rightarrow \mathrm{B}\left(\ell^{\hat{\otimes} n}\right)$. $\Lambda:=\bigoplus_{n=0}^{\infty} \Lambda^{(n)}: \mathcal{A} \rightarrow \mathrm{B}(\mathbf{L})$ is the direct sum Hilbert space representation of $\mathcal{A}$ on $\mathbf{L}$.

This justifies the following definition.

3.3 Definition. The operator norm $|a|:=\sup _{x \in \mathbf{L},\|x\|_{\mathbf{L}}=1}\|\Lambda(a) x\|_{\mathbf{L}}=\|\Lambda(a)\|_{\mathbf{L}}$ is a $C^{*}$-norm on $\mathcal{A}$. The corresponding completion of $\mathcal{A}$ is the $C^{*}$-algebra $\mathbf{A} . \Lambda(\mathbf{A})$ and $\mathbf{A}$ are identified.

3.4 Proposition. Comultiplication and counit on $\mathcal{A}$ are bounded and can be extended to continuous mappings on $\mathbf{A}, \Delta: \mathbf{A} \rightarrow \overline{\mathbf{A} \otimes \mathbf{A}} \underset{\text { closed }}{\subset} \mathrm{B}(\mathbf{L} \otimes \mathbf{L}), \epsilon: \mathbf{A} \rightarrow \mathbb{C}$.

Proof. The boundedness of $\epsilon$ follows from $|a|=\|\Lambda(a)\|_{\mathbf{L}} \geq\|\Lambda(a)\|_{\ell_{\hat{\otimes} 0}}=|\epsilon(a)|$. The boundedness of $\Delta$ comes from $\|(\Lambda \otimes \Lambda)(\Delta(a))\|_{\mathbf{L} \hat{\otimes} \mathbf{L}}=\|\Lambda(a)\|_{\mathbf{L}}$.

The antipode need not be continuous and can therefore not be extended on $\mathbf{A}$. If $\mathcal{A}$ is the linear span of the matrix elements of the unitary irreducible group representations of a compact topological group then $\mathbf{A}$ is the $C^{*}$-algebra of continuous functions over this group. This is the contents of the Peter-Weyl Theorem, see e. g. [3].

With the above construction the compact quantum group algebra $\mathcal{A}$ determines uniquely the $C^{*}$ completion A.

3.5 Lemma. The Haar functional $h$ is bounded on $\mathcal{A}$ respective to the norms $\|.\|_{\ell}$ and |.|. Its extension on $\ell$ is given by $\ell \ni x \mapsto\left(1_{\mathcal{A}} \mid x\right)$. Denoting the extension of $h$ on the $C^{*}$-algebra by $h: \mathbf{A} \rightarrow \mathbb{C}$ the following holds

$$
\forall a \in \mathbf{A}: h(a)=\left(1_{\mathcal{A}} \mid \Lambda(a) 1_{\mathcal{A}}\right)
$$

\section{Continuous correpresentations}

Now we can define continuous correpresentations of $\mathbf{A}$. Since $\sigma_{\mathrm{B}(L)}$ is bounded on $\mathrm{B}(\mathbf{L}) \otimes \mathrm{B}(\mathbf{L}) \subset$ $\mathrm{B}(\mathbf{L} \hat{\otimes} \mathbf{L})$ it can be continued on the closure.

4.1 Definition. A continuous correpresentation $(U, H)$ of $\mathbf{A}$ on the Hilbert space $H$ is given by

(i) $\quad U \in \overline{\mathbf{A} \otimes \mathrm{B}(H)} \underset{\text { closed }}{\subset} \mathrm{B}(\mathbf{L} \hat{\otimes} H)$, such that 
(ii) $U_{13} U_{23}=(\Delta \otimes i d)(U), U_{23}:=1_{\mathbf{A}} \otimes U, U_{13}:=\left(\sigma_{\mathbf{A}} \otimes i d\right)\left(U_{23}\right)$,

(iii) $\quad(\epsilon \otimes i d)(U)=1_{\mathrm{B}(H)}$.

Let $U^{*}$ denote the adjoint of $U$ in $\mathrm{B}(\mathbf{L} \hat{\otimes} H)$.

The continuous correpresentation $(U, H)$ of $\mathbf{A}$ is called isometric if $U^{*} U=1_{\mathbf{A}} \otimes 1_{\mathrm{B}(H)}$.

$(U, H)$ is called unitary if $U U^{*}=1_{\mathbf{A}} \otimes 1_{\mathrm{B}(H)}=U^{*} U$.

In the case of a commutative $*$-Hopf algebra $\mathcal{A}$ standing for the polynomials over a compact topological group $G$ this definition is equivalent to the statement that the mapping $G \times H \rightarrow H$, $(g, v) \mapsto U(g)(v)$ is a continuous representation of $G$ on $H$.

If $H$ is finite dimensional then $U \in \mathbf{A} \otimes \mathrm{B}(H)$. It is evident that every correpresentation of $\mathcal{A}$ on a Hilbert space is in particular a continuous correpresentation of $\mathbf{A}$. The definition 2.2 of invariant subspaces and irreducibility can be taken over to the case of $\mathbf{A}$.

4.2 Definition. Let $(U, H)$ be a continuous correpresentation of $\mathbf{A}$. The closed subspace $K \underset{\text { closed }}{\subset} H$ is called invariant if the orthogonal projector $P_{K} \in \mathrm{B}(H), P_{K} H=K$ fulfills

$$
U\left(1_{\mathbf{A}} \otimes P_{K}\right)=\left(1_{\mathbf{A}} \otimes P_{K}\right) U\left(1_{\mathbf{A}} \otimes P_{K}\right)=: U_{K}
$$

In this case $\left(U_{K}, K\right)$ is called a continuous subcorrepresentation of $\mathbf{A}$.

The continuous correpresentation $(U, H)$ is called irreducible if the only invariant subspaces of $H$ are $H$ and $\{0\}$.

Let $(U, H)$ and $(Q, N)$ be continuous correpresentations of $\mathbf{A}$ on the Hilbert spaces $H$ and $N$, let $L: H \rightarrow N$ be linear and continuous. If

$$
Q\left(1_{\mathbf{A}} \otimes L\right)=\left(1_{\mathbf{A}} \otimes L\right) U
$$

then $L$ is called an intertwiner.

These definitions are justified by the following observation which can easily be verified.

4.3 Lemma. The continuous subcorrepresentation $\left(U_{K}, K\right)$ is a continuous correpresentation. If $(U, H)$ is isometric then each continuous subcorrepresentation is also isometric.

4.4 Schur's Lemma. Let $L: H \rightarrow N$ be an intertwiner for the correpresentations $(U, H)$ and $(Q, N)$ of $\mathbf{A}$ on the Hilbert spaces $H$ and $N$ with closed image $L(H) \underset{\text { closed }}{\subset} N$. If both are irreducible then either $L=0$ or $L$ is bijective.

Proof. Define in $\mathrm{B}(H)$ the orthogonal projector $P_{0}, P_{0} H=k e r L$ and in $\mathrm{B}(K)$ the orthogonal projector $P_{1}, P_{1} N=L(H)$. Apply to equation (5) $1_{\mathbf{A}} \otimes P_{0}$ from the right or $1_{\mathbf{A}} \otimes P_{1}$ from the left respectively to prove that $\operatorname{ker} L$ and $L(H)$ are invariant. If $L \neq 0$ then $\operatorname{ker} L=\{0\}$ because of the irreducibility of $(U, H)$. If $H \neq\{0\}$ it then follows from the irreducibility of $(Q, N)$ that $N=L(H)$.

4.5 Lemma. Let $H$ be a finite-dimensional Hilbert space, $(U, H)$ an irreducible isometric continuous correpresentation of $\mathbf{A}$. 
(i) $\quad U \in \mathcal{A} \otimes \mathrm{B}(H)$, and $(U, H)$ is an irreducible correpresentation of $\mathcal{A}$.

(ii) $\quad(U, H)$ is unitary, $(S \otimes i d)(U)=U^{*}$.

Proof. Let $\left\{e_{A}\right\}_{A=1, . ., d}$ be an orthonormal basis in $H$, and $e_{A}^{*}:=\left(e_{A} \mid.\right)$ the dual basis. Expand $U=\sum_{A, B=1}^{d} u_{A B} \otimes\left(e_{A} \otimes e_{B}^{*}\right) \in \mathbf{A} \otimes \mathrm{B}(H)$. Consider the following matrices $M_{i A}^{(\alpha, j, B)}:=h\left(t_{j i}^{\alpha *} u_{B A}\right)$. There is at least one $\alpha_{u} \in \hat{\mathcal{A}}$ and $j, B$ such that $M^{\left(\alpha_{u}, j, B\right)} \neq 0$. The opposite would imply the contradiction

$$
\begin{aligned}
0 & =\sum_{B} \sum_{\alpha, i, j} h\left(u_{B A}^{*} t_{i j}^{\alpha}\right) \frac{1}{\left\|t_{i j}^{\alpha}\right\|_{\ell}^{2}} h\left(t_{i j}^{\alpha *} u_{B A}\right) \\
& =\sum_{B} \sum_{\alpha, i, j}\left(\Lambda\left(u_{B A}\right) 1_{\mathcal{A}} \mid t_{i j}^{\alpha}\right) \frac{1}{\left\|t_{i j}^{\alpha}\right\|_{\ell}^{2}}\left(t_{i j}^{\alpha} \mid \Lambda\left(u_{B A}\right) 1_{\mathcal{A}}\right) \\
& =\sum_{B}\left(\Lambda\left(u_{B A}\right) 1_{A} \mid \Lambda\left(u_{B A}\right) 1_{A}\right)=\sum_{B}\left(1_{\mathcal{A}} \mid \Lambda\left(u_{B A}^{*} u_{B A}\right) 1_{\mathcal{A}}\right)=1
\end{aligned}
$$

where Lemma 3.5 and the isometry of $(U, H)$ have been used. In $V^{\alpha_{u}}$ an orthonormal basis $\left\{f_{i}\right\}$ with $f_{i}^{*}:=\left(f_{i} \mid.\right)$ such that $T^{\alpha_{u}}=\sum_{i j} t_{i j}^{\alpha} \otimes\left(f_{i} \otimes f_{j}^{*}\right)$ is used. Then $L:=\sum_{i A} M_{i A}^{\left(\alpha_{u}, j, B\right)}\left(f_{i} \otimes e_{A}^{*}\right)$ is a nonvanishing intertwiner $\left(1_{\mathbf{A}} \otimes L\right) U=T^{\alpha_{u}}\left(1_{\mathbf{A}} \otimes L\right)$. Since $L(H)$ is closed Schur's Lemma implies that $L$ is invertible, and one gets $U=\left(1_{\mathcal{A}} \otimes L^{-1}\right) T^{\alpha_{u}}\left(1_{\mathcal{A}} \otimes L\right) \in \mathcal{A} \otimes \mathrm{B}(H)$ thus proving the first statement. Applying the antipode to the last equation one gets $(S \otimes i d)(U)=\left(1_{\mathcal{A}} \otimes L^{-1}\right) T^{\alpha_{u} *}\left(1_{\mathcal{A}} \otimes L\right)=U^{*}$ and hence the unitarity of $U$.

Statement (i) of Lemma 4. implies that the $*$-Hopf algebra can be uniquely reconstructed from its $C^{*}$-completion $\mathbf{A}$.

4.6 Proposition. Let $(U, H)$ be an isometric continuous correpresentation on the Hilbert space $H$, $K$ a finite dimensional invariant subspace such that the continuous subcorrepresentation $\left(U_{K}, K\right)$ is irreducible.

(i) $\left(U_{K}, K\right)$ is a unitary continuous correpresentation of $\mathbf{A}$.

(ii) $U\left(1_{\mathbf{A}} \otimes P_{K}\right)=\left(1_{\mathbf{A}} \otimes P_{K}\right) U$.

(iii) $\quad H \ominus K$ is invariant, $\left(\left(1_{\mathbf{A}} \otimes\left(1_{\mathrm{B}(H)}-P_{K}\right)\right) U, H \ominus K\right)$ is an isometric continuous correpresentation of $\mathbf{A}$.

(iv) If $H$ is finite dimensional then it decomposes in irreducible continuous subcorrepresentations, and $U \in \mathcal{A} \otimes \mathrm{B}(H)$.

Proof. The first statement follows from Lemmas 4.3 and 4.5. One gets therefore $1_{\mathbf{A}} \otimes P_{K}=$ $U_{K} U_{K}^{*}=U\left(1_{\mathbf{A}} \otimes P_{K}\right) U^{*}$. Multiplying with $U$ from the right proves the second statement. The third one is a direct consequence using Lemma 4.3. The last assertion follows by induction.

The last statement means that the finite dimensional unitary continuous correpresentations of $\mathbf{A}$ coincide with the finite dimensional unitary correpresentations of $\mathcal{A}$.

4.7 Definition. Let $f \in \mathcal{A},(U, H)$ an isometric continuous correpresentation of $\mathbf{A}$ on the Hilbert space $H$.

$$
h_{f}:=(h \otimes i d)\left(\left(f \otimes 1_{\mathrm{B}(H)}\right) U\right) \in \mathrm{B}(H) .
$$


4.8 Proposition. Let $(U, H)$ be an isometric continuous correpresentation of $\mathbf{A}$ on the Hilbert space $H$. Let $\left\{\left(U_{K_{\rho}}, K_{\rho}\right)\right\}_{\rho \in R}$ be a family of irreducible finite dimensional continuous subcorrepresentations, let $P_{\rho}:=P_{K_{\rho}}$ be the orthogonal projectors on $K_{\rho}$ respectively.

(i) The norm of $h_{f}$ is bounded by $\left\|h_{f}\right\|_{H} \leq\|f\|_{\ell}$.

(ii) $P_{\rho} h_{f}=h_{f} P_{\rho}$.

(iii) $\quad U\left(1_{\mathbf{A}} \otimes h_{f}\right)=\sum_{A} S\left(f_{A}^{\prime}\right) \otimes h_{f_{A}^{\prime \prime}}$ where $\Delta(f)=\sum_{A} f_{A}^{\prime} \otimes f_{A}^{\prime \prime}$.

(iv) If $v \neq 0$ then there is an $f \in \mathcal{A}$ such that $h_{f}(v) \neq 0$.

Proof. Apply $U$ to $1_{\mathcal{A}} \otimes v \in \ell \hat{\otimes} H \subset \mathbf{L} \hat{\otimes} H . U\left(1_{\mathcal{A}} \otimes v\right)=: \sum_{i} u_{i} \otimes v_{i} \in \ell \hat{\otimes} H$ such that the $v_{i} \in H$ are mutually orthogonal. Then $h_{f}(v)=\sum_{i}\left(f^{*} \mid u_{i}\right) v_{i}$. The first assertion follows from $\left\|h_{f}(v)\right\|_{H}^{2}=$ $\sum_{i}\left|\left(f^{*} \mid u_{i}\right)\right|^{2}\left\|v_{i}\right\|_{H}^{2} \leq \sum_{i}\left(f^{*} \mid f^{*}\right)\left(u_{i} \mid u_{i}\right)\left\|v_{i}\right\|_{H}^{2}=\left\|f^{*}\right\|_{\ell}^{2}\|v\|_{H}^{2}$. The second is obvious. $U\left(1_{\mathbf{A}} \otimes h_{f}\right)=$ $(i d \otimes h \otimes i d)\left(\left(1_{\mathbf{A}} \otimes f \otimes 1_{\mathrm{B}(H)}\right) U_{13} U_{23}\right)=(i d \otimes h \otimes i d) \sum_{A}\left(\left(\epsilon\left(f_{A}^{\prime}\right) 1_{\mathbf{A}} \otimes f_{A}^{\prime \prime} \otimes 1_{\mathrm{B}(H)}\right)(\Delta \otimes i d)(U)\right)$ proves the third statement. $h_{f}(v)=0$ is equivalent to $\left(f^{*} \mid u_{i}\right)=0$ for all $i$. Since $\mathcal{A}$ is dense in $\ell$ the forth statement follows.

4.9 Theorem. Let $(U, H)$ be an isometric continuous correpresentation of $\mathbf{A}$ on the Hilbert space $H$. Then $(U, H)$ is unitary and $H$ decomposes in finite dimensional irreducible continuous subcorrepresentations $\left(U_{\rho}, K_{\rho}\right), U_{\rho} \in \mathcal{A} \otimes \mathrm{B}\left(K_{\rho}\right)$ :

$$
H=\bigoplus_{\rho \in R} K_{\rho} .
$$

Proof. Using Zorn's Lemma let $R$ be a maximal index set such that $P_{\rho}$ with $\rho \in R$ are projectors on finite dimensional irreducible subspaces of $H$ with $P_{\rho} P_{\rho^{\prime}}=0$ if $\rho \neq \rho^{\prime}$. Then choose $v \in\left(1_{\mathrm{B}(H)}-\sum_{\rho \in R} P_{\rho}\right) H$. From proposition 4.8 it follows that $h_{f}(v)$ is contained in a finite dimensional continuous correpresentation. Hence $v$ is necessarily 0 . Since the restriction of $(U, H)$ to each irreducible continuous subcorrepresentation is unitary the full continuous correpresentation is necessarily unitary.

The theory of unitary continuous correpresentations of the $C^{*}$-completions of the $*$-Hopf algebras of compact quantum groups is thus reduced to the correpresentation theory of the underlying $*$-Hopf algebras. In [7] S. L. Woronowicz showed that the theory of finite dimensional correpresentations of the $*$-Hopf algebra of $S U_{q}(N)$ coincides with the theory for the undeformed $S U(N)$. Together with our results one concludes therefore that also the theory of unitary continuous correpresentations of these quantum groups coincides with the theory for the undeformed Lie groups.

The authors acknowledge gratefully discussions with M. Dijkhuizen, T. Koornwinder and J. Wess. 


\section{References}

[1] V. G. Drinfeld, Proceedings of the International Congress of Mathematicians, Berkeley, California, 798 (1986)

[2] L. D. Faddeev, N. Yu. Reshetikhin, L. A. Takhtajan, Algebra and Analysis 1, 178 (1989)

[3] A. W. Knapp, Lie groups, Lie algebras, and cohomology, Mathematical Notes 34, Princeton 1988

[4] T. H. Koornwinder, Lectures at the European School of Group Theory, Trento 1993 M. S. Dijkhuizen, Thesis, CWI Amsterdam 1993

[5] M. Rosso, Comm. Math. Phys. 117, 581 (1988)

[6] S. L. Woronowicz, Comm. Math. Phys. 111, 139 (1987)

[7] S. L. Woronowicz, Invent. math. 93, 35 (1988) 